\title{
Recurrent Muscle Cramps in a Boy
}

\author{
Adarsh Kancharla ${ }^{1} \cdot$ Ranjith Kumar Manokaran ${ }^{2}$ (])
}

Received: 27 May 2021 / Accepted: 17 August 2021 / Published online: 1 September 2021

(c) Dr. K C Chaudhuri Foundation 2021

To the Editor: Andersen-Tawil syndrome (ATS) is a clinical entity characterized by a classic triad of episodic flaccid muscle weakness (periodic paralysis), ventricular arrhythmia with prolonged QT interval, and skeletal anomalies [1]. It may occur sporadically or as an autosomal dominant trait. Hereby, we report an atypical presentation of Anderson-Tawil syndrome in a child.

An 8-y-old boy, born of a nonconsanguineous marriage, presented with recurrent episodes of fatigability and muscular cramps in both calves while playing. These episodes have been noticed from $5 \mathrm{y}$ of age. These episodes usually occurred in the late evening, while resting or after a heavy exercise. These episodes used to resolve in few days spontaneously. None of these episodes were associated with bulbar, facial, or respiratory muscle weakness, and sensory or bladder involvement. On examination, he was found to have bilateral mild calf hypertrophy. He did not have any dysmorphic features. Deep tendon reflexes were hypoactive. Gower sign was present. Investigations revealed mildly elevated serum creatine kinase (349 U/L). Nerve conduction studies were normal. Electromyography was not done. Serum electrolytes were normal. Mutiplex ligand probe amplification test for dystrophinopathies was negative.

Clinical exome sequencing showed a pathogenic heterozygous missense variation in exon 2 of $K C N J 2$ gene (chr17:g.70175691C>T) suggestive of ATS. Electrocardiogram did not show any evidence of long QT syndrome. Literature review suggests that this syndrome has variable expressivity [1]. Although ATS is characterized by episodes of muscle weakness, fixed myopathy like in our case has been reported only in 3 patients previously. All these 3 patients and our patient had heterozygous variationin inward rectifier potassium channel C-terminal domain of the $\mathrm{KCNJ} 2$ protein [2].

This report highlights that ATS may present with recurrent muscle cramps as a presenting symptom. Hence, this syndrome should also be considered as a differential diagnosis in a child presenting with muscle cramps.

\section{Declarations}

Conflict of Interest None.

\section{References}

1. Plaster NM, Tawil R, Tristani-Firouzi M, et al. Mutations in Kir2.1 cause the developmental and episodic electrical phenotypes of Andersen's syndrome. Cell. 2001;105:511-9.

2. ÖzTunçer G, Kutluk MG, Albayrak P, Teber S, Deda G. P1622473: Andersen-Tawil syndrome with myopathy: case report. Eur J of Paediatr Neurol. 2015;19:S139.

Publisher's Note Springer Nature remains neutral with regard to jurisdictional claims in published maps and institutional affiliations.
Ranjith Kumar Manokaran

doct.ranjith@gmail.com

1 Department of Pediatrics, Sri Ramachandra Institute of Higher Education and Research, Porur, Chennai, Tamil Nadu, India

2 Division of Pediatric Neurology, Department of Neurology, Sri Ramachandra Institute of Higher Education and Research, Porur, Chennai, Tamil Nadu 600116, India 